\title{
SIVA1 is a differentially expressed gene in brain metastatic human breast cancer.
}

Shahan Mamoor, $\mathrm{MS}^{1}$

${ }^{1}$ shahanmamoor@gmail.com

East Islip, NY USA

Metastasis to the brain is a clinical problem in patients with breast cancer ${ }^{1-3}$. We mined published microarray data ${ }^{4,5}$ to compare primary and metastatic tumor transcriptomes for the discovery of genes associated with brain metastasis in humans with metastatic breast cancer. We found that the apoptosis regulatory protein, Siva, encoded by SIVA1, was among the genes whose expression was most different in the brain metastases of patients with metastatic breast cancer as compared to primary tumors of the breast ${ }^{4}$. SIVA1 mRNA was present at increased quantities in brain metastatic tissues as compared to primary tumors of the breast. Importantly, expression of SIVA1 in primary tumors was significantly correlated with patient recurrence-free survival. Modulation of SIVA1 expression may be relevant to the biology by which tumor cells metastasize from the breast to the brain in humans with metastatic breast cancer.

Keywords: breast cancer, metastasis, brain metastases, central nervous system metastases, the apoptosis regulatory protein, Siva, SIVA1, systems biology of breast cancer, targeted therapeutics in breast cancer. 
One report described a $34 \%$ incidence of central nervous system metastases in patients treated with trastuzumab for breast cancer ${ }^{2}$. More recently, the NEfERT-T clinical trial ${ }^{6}$ which compared administration of either neratinib or trastuzumab in conjunction with paclitaxel demonstrated that in a randomized, controlled setting, in breast cancer patients treated with neratinib, not only was the incidence of central nervous system recurrence significantly lower, the time to central nervous system metastasis was significantly delayed as compared to patients administered trastuzumab ${ }^{6}$. The alarmingly high rate of central nervous system metastasis described, as well as data, both anecdotal ${ }^{2}$ and from a randomized, controlled setting ${ }^{6}$ illustrating $^{2}$ that treatment with trastuzumab may be associated with these events demands an enhanced understanding of the transcriptional makeup of brain metastatic tissues to support identification of therapeutic targets, whether they are treatment related or not. We performed a global comparative analysis of primary and metastatic tumors in patients with brain metastatic breast cancer ${ }^{4,5}$. We discovered differential and increased expression of the gene encoding the apoptosis regulatory protein, Siva, SIVA1, in brain metastatic tissues of patients with metastatic breast cancer.

\section{Methods}

We used datasets GSE10893 ${ }^{4}$ and GSE100534 ${ }^{5}$ for this global differential gene expression analysis of brain metastatic breast cancer in conjunction with GEO2R. GSE10893 was generated using Agilent-011521 Human 1A Microarray G4110A technology with $n=11$ primary breast tumors and $n=3$ brain metastases from patients with metastatic breast cancer; analysis was performed using platform GPL885. GSE100534 was generated using Affymetrix Human Gene 1.0 ST Array with $n=16$ primary breast tumors and $n=3$ brain metastases from patients metastatic breast cancer; analysis was performed using platform GPL6244. The Benjamini and Hochberg method of $p$-value adjustment was used for ranking of differential expression but raw $p$-values were used to assess statistical significance of global differential expression. Log-transformation of data was auto-detected, and the NCBI generated category of platform annotation was used. A statistical test was performed to evaluate whether SIVA1 gene expression was significantly different between primary tumors of the breast and brain metastases in humans with breast cancer using a two-tailed t-test. For Kaplan-Meier survival analysis, we used the Kaplan-Meier plotter online tool ${ }^{7}$ for correlation of SIVA1 mRNA expression levels with recurrence-free survival in $n=3951$ breast cancer patients, and with distant metastasis-free survival in $n=1803$ breast cancer patients.

\section{$\underline{\text { Results }}$}

We performed global comparative transcriptome analysis of metastatic tumor tissues of patients with metastatic breast cancer using published microarray data ${ }^{4,5}$ to describe the transcriptional landscape of brain metastasis in human breast cancer in an unbiased fashion and for the discovery of novel therapeutic targets.

\section{SIVA1 is differentially expressed in the brain metastases of patients with brain metastatic breast cancer.}

Through blind, systems-level analysis of published microarray data ${ }^{4}$, we identified the 
the apoptosis regulatory protein, Siva, encoded by SIVA1, as a differentially expressed gene in the breast metastatic tissues of humans with breast cancer (Table 1). When sorting each of the genes expressed in brain metastases based on significance of difference as compared to primary tumors of the breast in patients with breast cancer, SIVA1 ranked 65 out of 17418 total transcripts (Chart 1), equating to $99.6 \%$ differential expression. Differential expression of SIVA1 in the brain metastases of patients with metastatic breast cancer was statistically significant (Chart 1; $p=1.48 \mathrm{e}-04)$.

To attempt to validate transcriptome-wide differential expression of SIVA1 in brain metastatic tissues of patients with metastatic breast cancer, we queried a second microarray dataset $^{5}$, here comparing primary tumors from patients with breast cancer to brain metastases. Again, we identified SIVA1 as a differentially expressed gene in human brain metastatic breast cancer (Chart 2). When sorting each of the genes expressed in brain metastases based on significance of difference as compared to primary tumors of the breast, SIVA1 ranked 2109 out of 33297 total transcripts (Chart 2), equating to $93.7 \%$ differential expression. Differential expression of SIVA1 in the brain metastases of patients with metastatic breast cancer was statistically significant (Chart $2 ; p=1.69 \mathrm{e}-03$ ). Thus, differential expression of SIVA1, transcriptome-wide, in brain metastatic tissues of patients with metastatic breast cancer could be observed across two independent microarray datasets.

\section{SIVA1 is expressed at higher levels in the brain metastases of patients with metastatic breast} cancer.

We obtained exact mRNA expression levels for SIVA1, in primary tumors of the breast and in brain metastasis of patients with brain metastatic breast cancer to determine direction and statistical significance of change in SIVA1 expression in brain metastatic tissues. We observed significantly higher expression of SIVA1 in the brain metastases of patients with breast cancer as compared to primary tumors of the breast: SIVA1 was expressed at $-0.44 \pm 0.25$ arbitrary units (AU) in primary tumors of the breast, while it was expressed at $-0.03 \pm 0.89$ AU in brain metastatic tissues (Figure 1). The difference in SIVA1 mRNA levels between primary tumors of the breast and brain metastatic tissues was statistically significant (Figure $1 ; p=0.000293$ ).

SIVA1 expression is significantly correlated with survival outcomes in human breast cancer.

We performed Kaplan-Meier survival analysis ${ }^{7}$ in 3951 breast cancer patients in total, to evaluate whether SIVA1 tumor expression was correlated with survival outcomes in human breast cancer. We observed a statistically significant correlation between primary tumor expression of SIVA1 and recurrence-free survival (RFS) in patients with breast cancer, in the upper survival quartile (Figure 2). Patients whose primary tumors expressed low levels of SIVA1 possessed median RFS of 55 months, while patients whose tumors expressed high levels of SIVA1 possessed median RFS of 35 months. This difference in RFS based on SIVA1 tumor expression in patients with breast cancer was statistically significant (Figure 2, Chart 3; logrank $p$-value: 1.2e-06; hazard ratio: 1.34 (1.19-1.51)). 
Thus, by mining published microarray data ${ }^{4,5}$ in an unbiased and systematic fashion, we identified the apoptosis regulatory protein, Siva, encoded by SIVA1, as among the genes whose expression was most different, transcriptome-wide, in the brain metastases of patients with breast cancer, when compared to primary tumors of the breasts; we observed significantly increased expression of SIVA1 in brain metastases as compared to primary tumors of the breast. Further, we found a significant correlation between SIVA1 expression and patient survival outcomes, as recurrence-free survival was significantly greater in patients whose primary tumors expressed lower levels of SIVA1 as compared to patients whose primary tumors expressed higher levels of SIVA1.

\section{Discussion}

We provided evidence here that the the apoptosis regulatory protein, Siva, encoded by SIVA1, is among the genes whose expression is most different in the brain metastases of patients with brain metastatic breast cancer, that SIVA1 mRNA is present at significantly increased quantities in brain metastatic tissues as compared to primary tumors of the breast, and that primary tumor SIVA1 expression is significantly correlated with patient survival outcomes in human breast cancer. Evaluation of the effects of genetic depletion of SIVA1 in mouse models of metastatic breast cancer on metastasis to the central nervous system is merited. Modulation of SIVA1 expression may be relevant to the processes by which breast cancer cells exit the breast, enter the vasculature and/or lymphatics, reside in the lymph nodes, evade immune clearance, breach the blood-brain barrier and colonize the brain. 


\section{References}

1. Lin, N.U., Amiri-Kordestani, L., Palmieri, D., Liewehr, D.J. and Steeg, P.S., 2013. CNS metastases in breast cancer: old challenge, new frontiers.

2. Bendell, J.C., Domchek, S.M., Burstein, H.J., Harris, L., Younger, J., Kuter, I., Bunnell, C., Rue, M., Gelman, R. and Winer, E., 2003. Central nervous system metastases in women who receive trastuzumab-based therapy for metastatic breast carcinoma. Cancer, 97(12), pp.2972-2977.

3. Tsukada, Y., Fouad, A., Pickren, J.W. and Lane, W.W., 1983. Central nervous system metastasis from breast carcinoma autopsy study. Cancer, 52(12), pp.2349-2354.

4. Weigman, V.J., Chao, H.H., Shabalin, A.A., He, X., Parker, J.S., Nordgard, S.H., Grushko, T., Huo, D., Nwachukwu, C., Nobel, A. and Kristensen, V.N., 2012. Basal-like Breast cancer DNA copy number losses identify genes involved in genomic instability, response to therapy, and patient survival. Breast cancer research and treatment, 133(3), pp.865-880.

5. Schulten, H.J., Bangash, M., Karim, S., Dallol, A., Hussein, D., Merdad, A., Al-Thoubaity, F.K., Al-Maghrabi, J., Jamal, A., Al-Ghamdi, F. and Choudhry, H., 2017. Comprehensive molecular biomarker identification in breast cancer brain metastases. Journal of translational medicine, 15(1), p.269.

6. Awada, A., Colomer, R., Inoue, K., Bondarenko, I., Badwe, R.A., Demetriou, G., Lee, S.C., Mehta, A.O., Kim, S.B., Bachelot, T. and Goswami, C., 2016. Neratinib plus paclitaxel vs trastuzumab plus paclitaxel in previously untreated metastatic ERBB2-positive breast cancer: the NEfERT-T randomized clinical trial. JAMA oncology, 2(12), pp.1557-1564.

7. Györffy, B., Lanczky, A., Eklund, A.C., Denkert, C., Budczies, J., Li, Q. and Szallasi, Z., 2010. An online survival analysis tool to rapidly assess the effect of 22,277 genes on breast cancer prognosis using microarray data of 1,809 patients. Breast cancer research and treatment, 123(3), pp.725-731. 


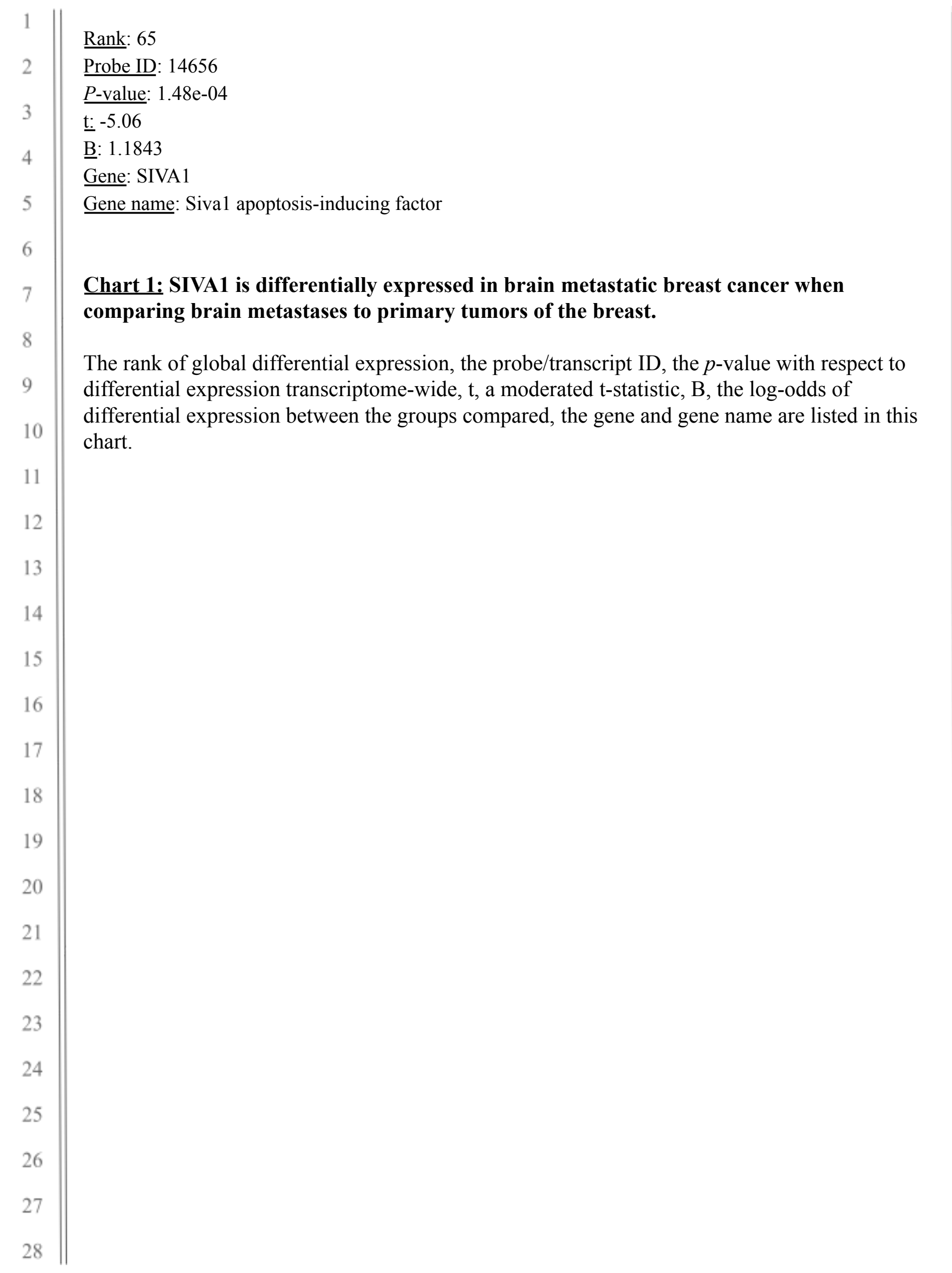




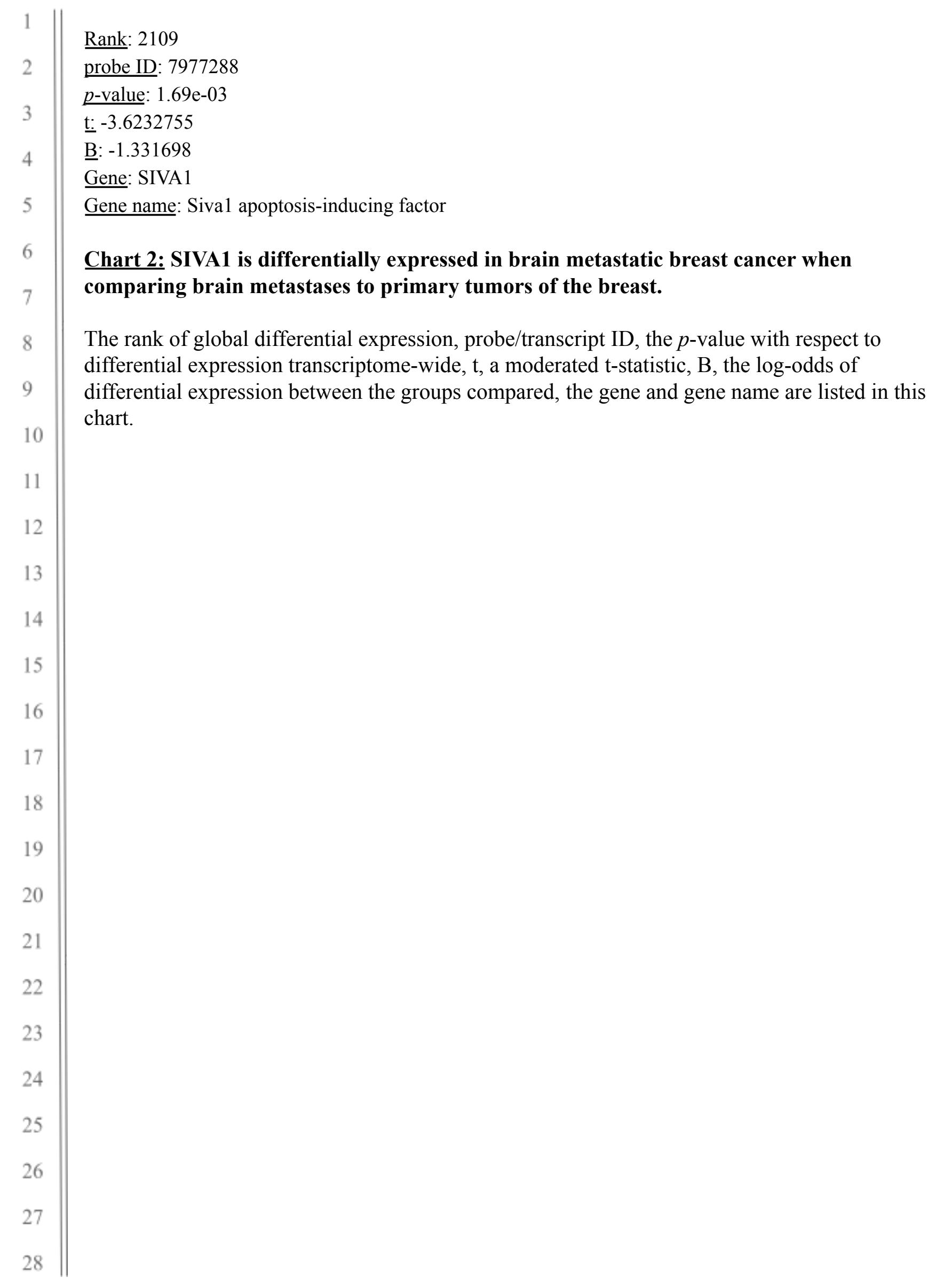




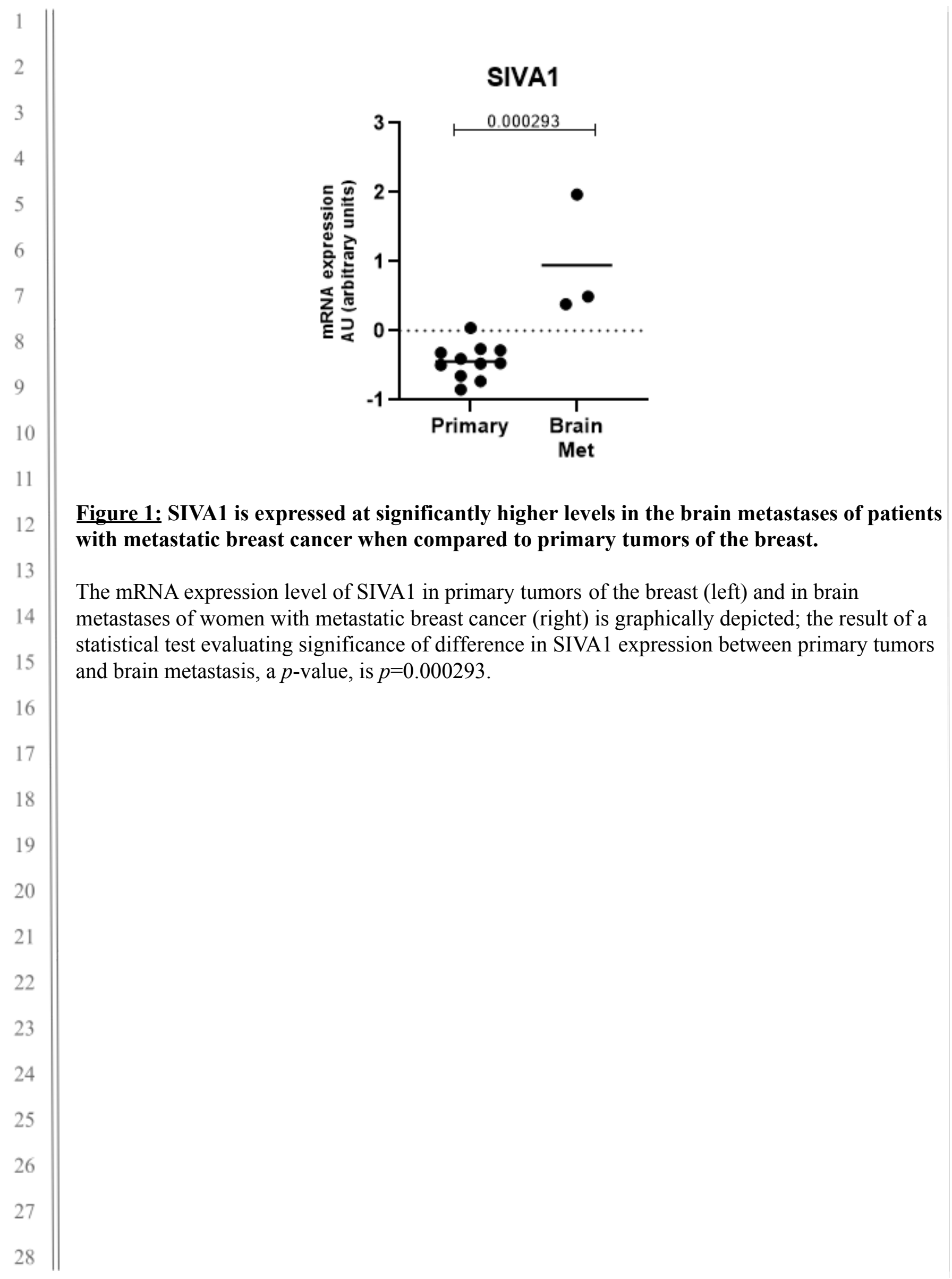




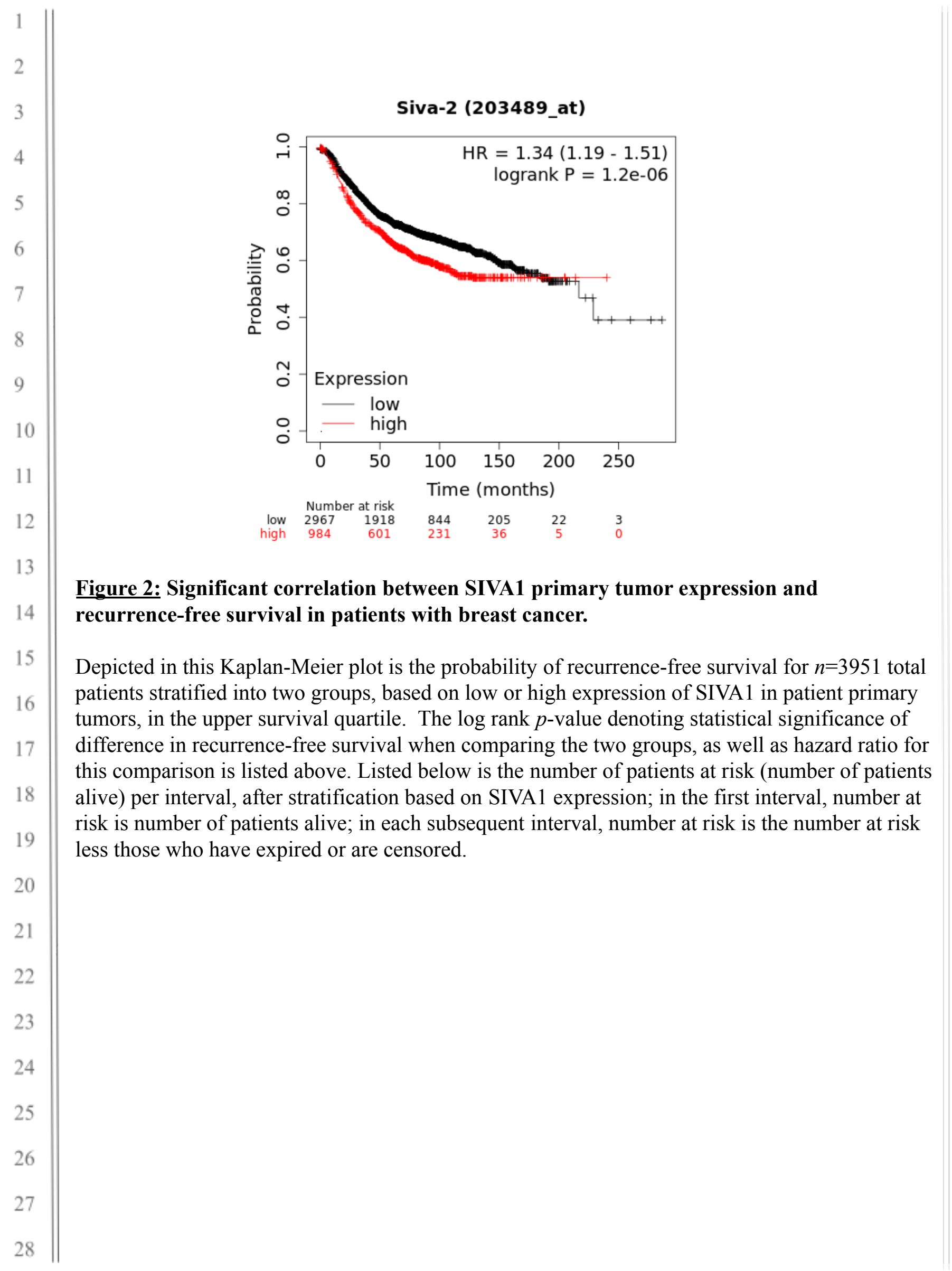


Low SIVA1 expression: 55 months

High SIVA1 expression: 35 months

Chart 3: Median recurrence-free survival is inferior in patients with high primary tumor expression of SIVA1.

The median RFS (recurrence-free survival) of patients with low primary tumor expression of

6 SIVA1 and high primary tumor expression of SIVA1 is listed in this chart, for $n=3951$ breast cancer patients, in the upper survival quartile. 